\title{
Pueraria montana var. lobata Root Extract Inhibits Photoaging on Skin through Nrf2 Pathway
}

\author{
Hee Sun Heo, Ga Eun Han, Junho Won, Yeonoh Cho, Hyeran Woo, and Jong Hun Lee \\ Department of Food Science and Biotechnology, College of Life Sciences, CHA University, Gyeonggi-do 11160, Republic of Korea
}

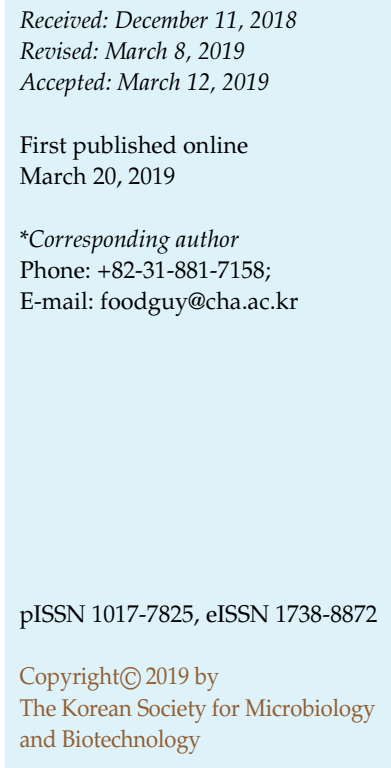

Pueraria montana var. lobata is a bioactive substance with various beneficial health effects and has long been extensively used as a traditional medication for the treatment of fever, acute dysentery, diabetes, and cardiovascular diseases in Northeast Asian countries. The purpose of this study was to evaluate the cytoprotective activity of Pueraria montana var. lobata ethanol extract (PLE) for ultraviolet B (UVB)-induced oxidative stress in human dermal fibroblasts (HDF). It was hypothesized that PLE treatment $(25-100 \mu \mathrm{g} / \mathrm{ml})$ would reduce intracellular reactive oxygen species (ROS) levels as well as increase collagen production in UVB-irradiated HDF. The results confirmed this theory, with collagen production increasing in the PLE treatment group in a dose-dependent manner. In addition, regulators of cellular ROS accumulation, including HO-1 and NOQ-1, were activated by Nrf2, which was mediated by PLE. Hence, intracellular levels of ROS were also reduced in the PLE treatment group in a dose-dependent manner. In conclusion, PLE increases collagen production and maintains hyaluronic acid (HA) levels in human dermal fibroblasts exposed to UVB-irradiation, thereby inhibiting photoaging.

Keywords: Pueraria montana var. lobata, UVB, photoaging, Nrf2

\section{Introduction}

There are two main types of skin aging; intrinsic aging and extrinsic aging [1]. In relation to the former, intrinsic skin aging presents as a decrease in the thickness of the epidermis and dermis [2], with overall levels of collagen and elastin diminishing, coupled with a concurrent increase in the quantity of collagen cross-linked fibers [3, 4]. Intrinsic aging will typically be observed in the form of only light wrinkles [2, 5], and is not associated with increased pigmentation or deep wrinkles [6]. By contrast, extrinsic aging, also referred to as 'photoaging' [2], can be found with clinical presentations which include skin dryness, sallowness, laxity, atrophy, elastosis, mottled pigmentation, and deep coarse wrinkles [7]. In photoaging, irradiation with UVB increases the generation of reactive oxygen species (ROS), which serve to increase the breakdown of collagen [8]. This occurs when the UV irradiation induces AP-1, which increases reactive oxygen species production and results in subsequent collagen breakdown [9]. At the same time, matrix metalloproteinase-1 (MMP-1) production increases [7, 10], as the oxidative stress triggers MMP secretion from dermal fibroblasts [11]. MMP-hydrolyzed extracellular matrix (ECM) proteins include collagen fibers. MMP-1 is important for initiating the breakdown of type I collagen and, in particular, yielding the degradation of ECM [10]. UVB absorbed by skin molecules produces harmful substances, ROS, which cause oxidative damage to cellular components such as cell walls, lipids, mitochondria, and DNA $[7,12,13]$. Moreover, in addition to metabolism and other biochemical reactions in live cells, ROS can be continuously produced as a result of external factors [14].

Nuclear factor-erythroid 2-related factor-2 (Nrf2) is critical for regulating cellular defense responses when cells are under oxidative stress [15]. In other words, Nrf2 is a redox-sensitive transcription factor that regulates the expression of a variety of antioxidant enzymes. Nrf2 is usually present in the cytoplasm, in an inactive complex with Kelch-like ECH-associated protein 1 (Keap1) [15, 16]. However, when activated it dissociates from Keap1 and 
translocates to the nucleus [15-17]. Nrf2 then binds to the antioxidant response element (ARE) to increase the expression of antioxidant enzymes, such as heme oxygenase 1 (HO-1), and $\mathrm{NAD}(\mathrm{P}) \mathrm{H}$ : quinone oxidoreductase 1 (NQO1) $[18,19]$. One of the enzymes up-regulated by Nrf2, HO-1, has both powerful antioxidant and anti-inflammatory properties, while also possessing the ability to catalyze the rate limiting reaction in heme degradation [19-21]. The expression of NQO1, another downstream enzyme, is very strongly induced by free radicals generated by UVB irradiation [22].

Pueraria montana var. lobata, also widely known as Pueraria lobata has long been used medicinally in Northeast Asian countries such as Japan, China, and Korea. Pueraria montana var. lobata is known as 'kudzu' in Japan, while the roots of Pueraria montana var. lobata are included in the Chinese Pharmacopoeia ('Gegen'; Radix Puerariae montana var. lobataa). In Korea it has been utilized under the name 'kalgeun.' The genus Pueraria is a rich source of isoflavones, the most abundant isoflavonoid in the root of Pueraria montana var. lobata. It has been known to provide relief for fever, dysentery, and cardiovascular diseases such as hypertension, myocardial infarction and arrhythmia [23].

This study was performed to empirically investigate the effects of Pueraria montana var. lobata extract (PLE) on the production of type I procollagen in UVB-irradiated HDFs, to determine the underlying antioxidant mechanisms, and ultimately to elucidate the inhibition effect of PLE on photoaging.

\section{Materials and Methods}

\section{Chemicals and Reagents}

The fetal bovine serum (FBS) and Dulbecco's Modified Eagle's Medium (DMEM) used in this study were purchased from Corning (USA). Penicillin-Streptomycin (PS) was acquired from Gibco (USA). Primary antibodies against $\beta$-actin (sc-1616), Nrf2 (sc-722), HO-1 (sc-10789), NQO-1 (sc-32793) and Keap-1 (sc-15246) were procured from Santa Cruz Biotechnology (USA). Primary antibodies against COL1A1 (ab34710) and MMP-1 (ab38929) were sourced from Abcam (USA). For secondary antibodies, donkey anti-goat immunoglobulin $\mathrm{G}$ (IgG)-horseradish peroxidase (HRP) (GTX232040-01) and rabbit IgG antibody HRP (GTX213110-01) were obtained from Gene Tex (Insight Biotech, UK). Lastly, goat anti-mouse-IgG-HRP conjugate (W 402B) was purchased from Progema (USA).

\section{Preparation of PLE}

The PLE used in this study was kindly provided by SCM Tech (Incheon, Namdong-gu, Korea). Fully-dried roots of Pueraria montana var. lobata were collected and powdered using an electric grinder. One hundred grams of powdered material was kept in $1 \mathrm{~L}$ of $70 \%$ ethanol solution and incubated at $60^{\circ} \mathrm{C}$ in a reciprocating shaker for $24 \mathrm{~h}$. Then, the extract was filtered by Whatman no. 1 filter paper with final filtration utilizing a vacuum pump. The solvent from the extract was removed via a rotary vacuum evaporator. Finally, the residues were collected and made use of in the experiments.

\section{Cell Culture and UVB-Irradiation}

Human dermal fibroblasts (HDFs) were obtained from ATCC (pcs-201-010) and prepared in accordance with the manufacturers' instructions. HDFs were cultured in DMEM containing 10\% FBS and $1 \%$ PS under a humidified atmosphere of $5 \% \mathrm{CO}_{2}$ at $37^{\circ} \mathrm{C}$. Human dermal fibroblasts from the $6^{\text {th }}$ to the $10^{\text {th }}$ passage were used for assays. Cells, at $1 \times 10^{6}$ cells $/ 10 \mathrm{~cm}$, were seeded in culture dishes.

Cell culture experiments were conducted $24 \mathrm{~h}$ after seeding. Irradiation was performed once, $24 \mathrm{~h}$ post cell culturing. Prior to UVB irradiation, the cells were rinsed twice with sterile phosphate-buffered saline (PBS). The cells were exposed to UVB radiation under a thin film of PBS without culture dish lids. Cells were irradiated with UVB at a dose of $20 \mathrm{~mJ} / \mathrm{cm}^{2}$ using a UVB CL1000 ultraviolet cross-linker (UVP Inc, USA). Immediately thereafter the medium was replaced.

\section{Cell Viability Assay}

Cell viability was assessed with a 3-(4,5-dimethylthiazol-2-yl)-5(3-carboxymethoxy-phenyl)-2-(4-sulfophenyl)-2H-tetrazolium (MTS) reagent, in accordance with the manufacturer's instructions. Human dermal fibroblasts were plated in 96-well culture plates at a density of $1 \times 10^{4}$ cells/well for $24 \mathrm{~h}$. After $24 \mathrm{~h}$ incubation, cells were treated with various concentrations of $\operatorname{PLE}(25,50$, and $100 \mu \mathrm{g} / \mathrm{ml}$ ) for $24 \mathrm{~h}$. After $24 \mathrm{~h}$ incubation, the cell medium was replaced with $15 \mu \mathrm{l}$ MTS reagent in $100 \mu \mathrm{l}$ DMEM and incubated at $37^{\circ} \mathrm{C}$ with $5 \% \mathrm{CO}_{2}$ for $2 \mathrm{~h}$. Absorbance at $490 \mathrm{~nm}$ was measured using a microplate reader (Biotek, USA).

$$
\text { Cell Survival Rate }=\left(\frac{\text { Absorbance in PLE treated group }}{\text { Absorbance in control group }}\right) \times 100 \%
$$

\section{Measurement of Intracellular ROS Production}

Cells were plated at $1 \times 10^{6}$ cells/well in $60 \mathrm{~mm}$ culture dishes with DMEM containing 10\% FBS and 1\% PA for $24 \mathrm{~h}$. Compounds were treated at $24 \mathrm{~h}$, using serum free media, before UVB irradiation. After triplicate washings with PBS, cells were irradiated with UVB at a dose of $20 \mathrm{~mJ} / \mathrm{cm}^{2}$, without lids. After being exposed to UVB for $24 \mathrm{~h}$, cells were incubated with $10 \mu \mathrm{M}$ of $2^{\prime}, 7^{\prime}$-dichlorofluorescein diacetate $\left(\mathrm{H}_{2} \mathrm{DCFDA}\right)$ (D399; Invitrogen, USA) in culture media at $37^{\circ} \mathrm{C}$ for $30 \mathrm{~min}$. Culture media were then removed and the pellets were suspended in $500 \mu \mathrm{l}$ PBS. Transference to $5 \mathrm{ml}$ FACS tube (SPL Life Sciences, Korea) then occurred. The cell suspension was examined by a FACSCalibur flow cytometer (Becton Dickinson, USA) equipped with a Nikon bandpass filter with an excitation wavelength of $488 \mathrm{~nm}$ and 
emission wavelength of $530 \mathrm{~nm}$. Data analyses were based on 10,000 detected events using Cell Quest software (USA).

\section{Measurement of Total Collagens}

A Sircol soluble collagen assay kit (Biocolor Ltd., UK) was used to quantify total soluble collagens in culture medium. Collected culture medium was centrifuged at $13,000 \mathrm{~g}$ for $10 \mathrm{~min}$ to drop the ECM, followed by the mixing of $100 \mu \mathrm{l}$ supernatant with $1 \mathrm{ml}$ of Sircol dye for $30 \mathrm{~min}$ and centrifuging at $13,000 \mathrm{~g}$ for $10 \mathrm{~min}$ to drop the formed collagen-dye complex. After removing the suspension, the pellets were dissolved in $250 \mu \mathrm{l}$ Sircol alkali reagent and vortexed. Relative absorbance was measured at $555 \mathrm{~nm}$ using a microplate reader (Biotek). The collagen content was calculated from a standard curve of the measured absorbance.

\section{Hyaluronic Acid Assay}

The Hyaluronan Quantikine ELISA kit (R\&D Systems, USA) was used to analyze the hyaluronic acid (HA) concentration of the supernatants of the HDF culture media. At near confluence, $50 \mu \mathrm{l}$ aliquots of the HDF cell culture supernatants were prepared and $50 \mu \mathrm{l}$ aliquots of the Assay Diluent RD1-14 were added to each well, as were, subsequently, the samples and then the buffer from the kit. Finally, stop solution was added, and the relative absorbance was measured at $540 \mathrm{~nm}$. The HA concentration was calculated from a standard curve of the measured absorbance.

\section{Western Blot Analysis}

After being exposed to UVB for $1 \mathrm{~h}$, the cell pellets were collected. Human dermal fibroblasts cells were lysed in $60 \mu \mathrm{l}$ of NP40 (Invitrogen, USA) containing protease inhibitors and then incubated on ice for 30 to $40 \mathrm{~min}$. Protein concentrations of whole cell lysates were measured using bicinchoninic acid (BCA) protein assay kits (Thermo Fisher Scientific, USA). Equal concentrations of protein $(12 \mu \mathrm{g})$ were separated by sodium dodecyl sulfate polyacetylamide gel electrophoresis (SDS-PAGE) and transferred to polyvinylidene fluoride (PVDF) membranes (Merck Millipore, Germany). The membranes were blocked with $5 \%$ bovine serum albumin (BSA) in Tris-buffered saline (TBST) containing 0.5\% tween-20 (TBST) for $1 \mathrm{~h}$ at room temperature (RT). After blocking, incubation with 1:1000 dilution of primary antibodies for $4 \mathrm{~h}$ at $\mathrm{RT}$, or $16 \mathrm{~h}$ at $4^{\circ} \mathrm{C}$, occurred. The membranes were washed in triplicate in TBST for $15 \mathrm{~min}$. Then they were incubated with secondary antibodies for $1 \mathrm{~h}$ at RT. After washing three times with TBST, separated protein bands were detected with an ImageQuant LSA 4000 (GE Healthcare, USA) using ECL solution (Dyne, Korea).

\section{Immunocytochemistry}

Cells were seeded at $3 \times 10^{4}$ cells/well in sterile 4-chamber slides (Lab-Tek, Thermo Fisher Scientific, USA) with DMEM containing 10\% FBS and 1\% PS for $24 \mathrm{~h}$. Cells were treated with compounds in serum-free DMEM for $24 \mathrm{~h}$ before UVB irradiation. After treatment, cells were washed in triplicate with PBS, then irradiated with UVB at a dose of $20 \mathrm{~mJ} / \mathrm{cm}^{2}$ without lids.
Immediately after irradiation, cells were given fresh media containing 10\% FBS and 1\% PS. After $1 \mathrm{~h}$ incubation, the cells were co-stained for COL1A1, antioxidant marker NQO1, and cell nuclei with 4',6-diamidino-2-phenylindole. Images were taken using an LSM 880 confocal laser scanning microscope (Zeiss, Germany) and processed with Zen black/blue software (Zeiss).

\section{Statistical Analysis}

All experiments were performed in triplicate, and all values are expressed as mean \pm standard error (SE) for $n=3$. Data were compared using the student's t-test. All analyses were conducted using SAS statistical software, version 9.1 (SAS Institute Inc, USA), and all statistical tests were two-sided, with statistical significance determined as $p$-value $<0.05$.

\section{Results and Discussion}

\section{Cytotoxicity of PLE on HDF}

The results of the MTS assay on HDF are illustrated in Fig. 1. The cells were treated with various concentrations ranging from 5 to $100 \mu \mathrm{g} / \mathrm{ml}$, the same concentrations used in the experiments performed using common extract. HDF did not exhibit a significant cytotoxicity, even at a very high concentration of $100 \mu \mathrm{g} / \mathrm{ml}$. The cells demonstrated a little less than $10 \%$ cytotoxicity at all the treated concentrations of PLE, except at a concentration of $5 \mu \mathrm{g} / \mathrm{ml}$. As shown in the cytotoxicity results, PLE was not shown to have a significant effect on cell viability. Thus, it was decided that three concentrations; 25, 50, and $100 \mu \mathrm{g} / \mathrm{ml}$, were to be used in this study.

\section{Effect of PLE on Collagen Production}

To determine the effect of PLE on collagen production, total soluble collagen levels and cellular collagen type 1

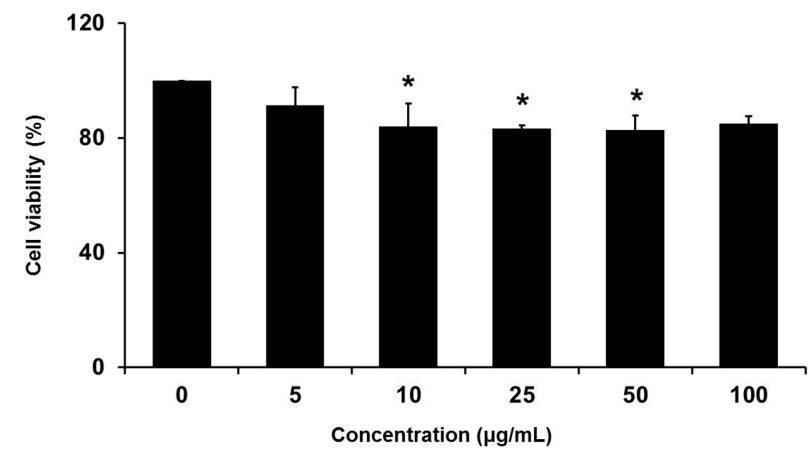

Fig. 1. Cell viability of HDF treated with various concentrations of Pueraria lobata extract (PLE) was assessed by MTS assay. Data represent the mean \pm standard error $(n=3)$. Asterisks indicate significant $(p<0.05)$ decreases in cell viability in comparison with non-treated group $(0 \mu \mathrm{g} / \mathrm{ml})$. 

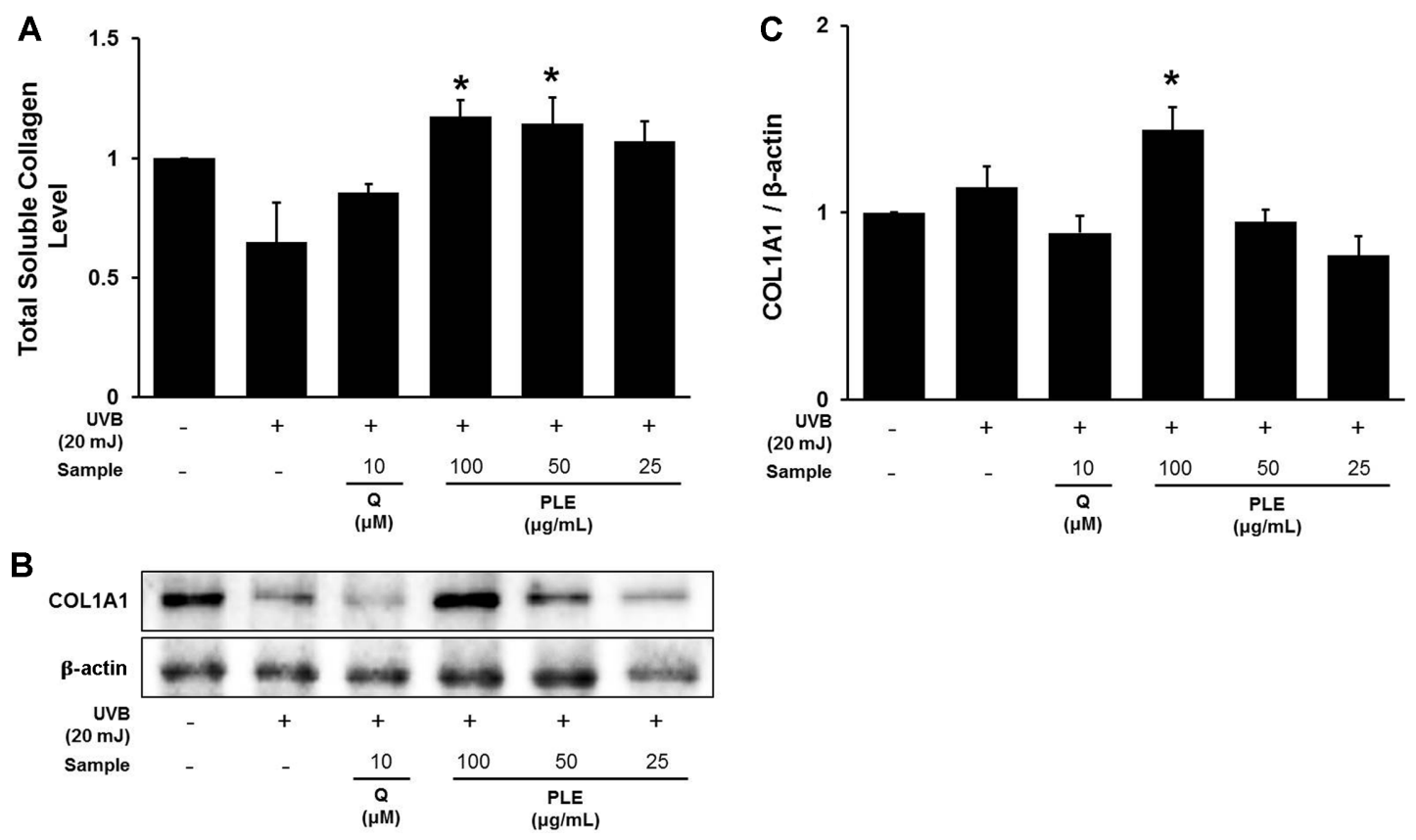

Fig. 2. Effect of PLE on collagen production was measured in UVB irradiated-HDF.

Quercetin (Q) was used as a positive control. UVB group was irradiated only with $20 \mathrm{~mJ} / \mathrm{cm}^{2}$ of UVB; Q group was treated with quercetin as a positive control, and the experimental groups were treated with the compound $24 \mathrm{~h}$ before UVB irradiation. (A) Total soluble collagens were determined in culture medium after $48 \mathrm{~h}$ of culture. (B) Type 1 collagen expression was measured using western blotting. Data represent the mean \pm standard error $(n=3)$. Asterisks indicate significant $(p<0.05)$ increases in collagen level compared with the UVB-treated group $(0 \mu \mathrm{g} / \mathrm{ml})$.

levels were measured using a Sircol soluble collagen assay kit and western blotting, respectively. According to the experimental results, increases in both the total soluble collagen and the collagen type 1 expression levels were observed in the ethanol extract treatment group, but the difference was not statistically significant (Fig. 2). The quantity of collagen in HDF was reduced by UVB irradiation, an effect that was recoverable by quercetin or PLE treatment. In the case of the PLE-treated group, total soluble collagen synthesis increased in a dose-dependent manner (Fig. 2A). Because type 1 collagen contributes to almost $80 \%$ of adult human dermis [24], it is important to measure the amount of type 1 collagen as well as the amount of total soluble collagen. Type I collagen, found in extensible connective tissue, such as the skin, respiratory, and vascular systems, is a fibrillar collagen that plays an important role in skin elasticity [8]. The effects of PLE on type 1 collagen expression were assessed. PLE increased type I collagen production in a dose-dependent manner in HDF (Figs. 5B and 5C).

Thus, these results demonstrate that UVB irradiation decreases collagen levels and that PLE treatment can restore not only total collagen levels, but also type 1 collagen expression, suggesting that PLE can recover UVBinduced collagen damage in HDF.

\section{Effect of PLE on Cellular Hyaluronic Acid Synthesis}

Hyaluronic acid (HA) is a polymer substance that forms the structure of cells. It is a polar molecule that contains moisture that helps preserve the structure of skin. In the present study, the production of HA was observed in HDF. Interestingly, among the experimental groups, only the quercetin $(\mathrm{Q})$ group (positive control) showed a statistically significant decrease in the production of HA. All other groups; the UVB group irradiated with UVB, the control (C) group which had no treatment, and the experimental group treated with PLE, exhibited no significant changes (Fig. 3). Moreover, in the PLE-treated group, the production of HA did not alter regardless of concentration changes in PLE. It can be deduced that UVB irradiation does not significantly affect the production of HA in HDF, as the amount of HA produced in the UVB treatment group did not decrease. Since the cells maintain an appropriate amount of HA, PLE does not excessively increase the 


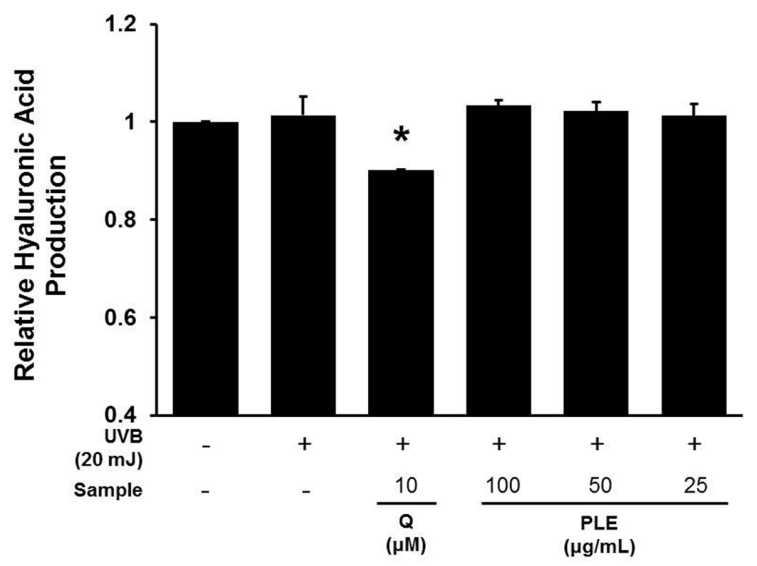

Fig. 3. Effect of PLE on relative HA production was estimated using ELISA.

HA concentration of the supernatants of HDF culture medium was measured at $540 \mathrm{~nm}$. Quercetin was used as a positive control. Data represent the mean \pm standard error $(n=3)$. Asterisk indicates significant $(p<0.05)$ changes in HA production compared with the UVB-treated group $(0 \mu \mathrm{g} / \mathrm{ml})$.

amount of HA in the cells. The reduction in HA observed in the quercetin-treated group can be explained by the quercetin inducing a change in the membranous structure of the cells [25].

\section{Inhibition of Intracellular ROS Production of PLE}

The intracellular ROS measurements were based on the principle that green fluorescence is generated when ROS, which increases by metabolism in the cells, reacts with $\mathrm{H}_{2}$ DCFDA. HDF was cultured in DMEM, and then incubated with PLE for $24 \mathrm{~h}$. After UVB irradiation $\left(20 \mathrm{~mJ} / \mathrm{cm}^{2}\right)$, intracellular ROS levels were measured by FACS. As shown in Fig. 4, it was observed that ROS in the UVB group, which was irradiated by UVB with no other treatment, accumulated at a relatively high concentration. However, in the experimental group treated with PLE extract, a decrease in the intracellular ROS level could be observed. The changes, in the intracellular ROS level in accordance with treatment concentrations of PLE, might confirm the inhibition effect of PLE on ROS production in HDF.

During photoaging, oxidative damage is induced by UVB irradiation, which increases collagen breakdown [8]. Thus, reduction of ROS generation can be an effective approach for the prevention of cellular damage caused by photoaging.
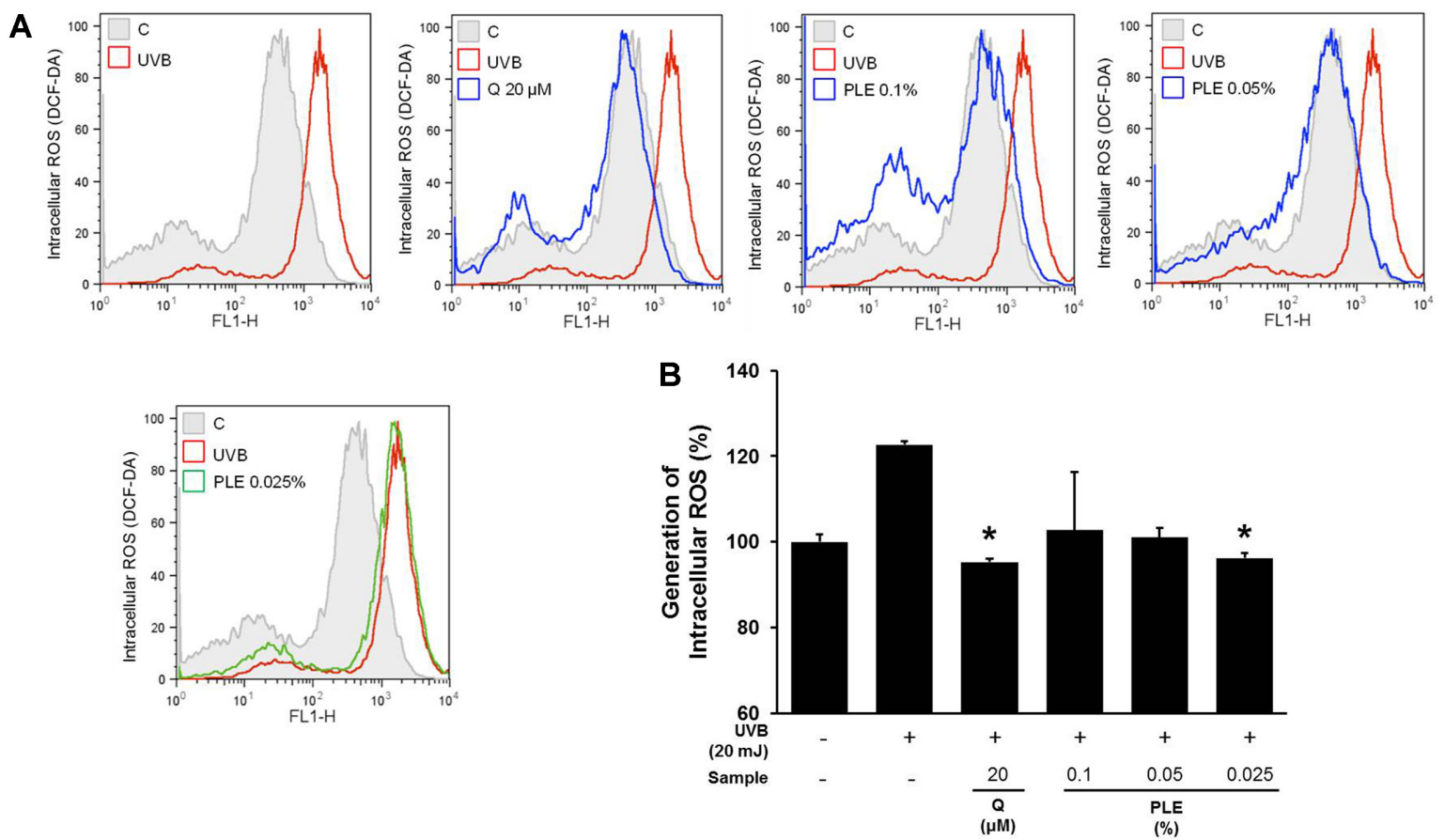

Fig. 4. Intracellular ROS levels were estimated by flow cytometric analysis of DCF fluorescence after staining cells with DCFDA. (A) Flow cytometric distribution of DCFDA-stained HDF. (B) Cell granularity of DCF-fluorescence was evaluated. Data analyses were based on 10,000 detected events using the Cell Quest software. Data represent the mean \pm standard error; asterisks indicate significant $(p<0.01)$ changes in ROS reduction as compared to UVB-treated group $(0 \mu \mathrm{g} / \mathrm{ml})$. PLE, Pueraria lobata extract treatment group cultured in medium; $\mathrm{Q}$, quercetin group used as a positive control. 
A

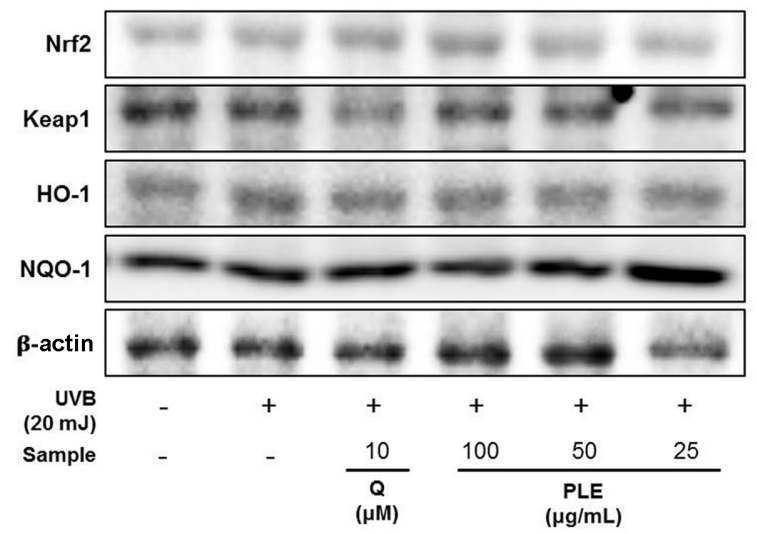

B

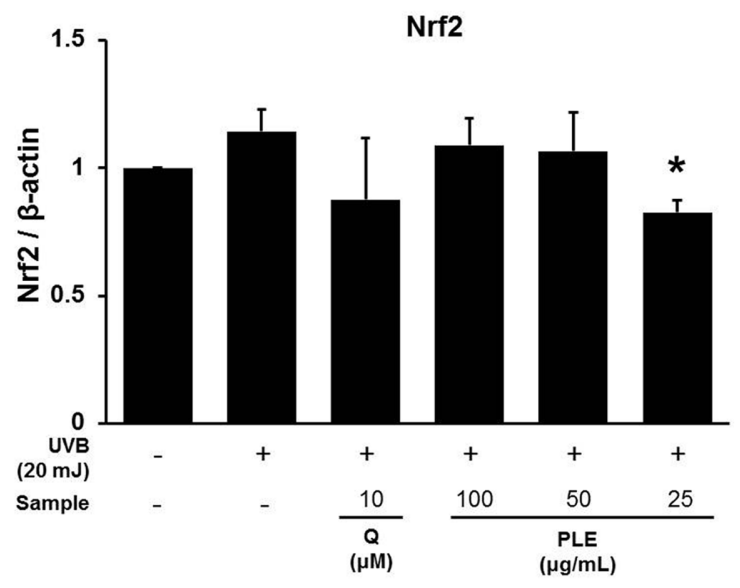

HO-1

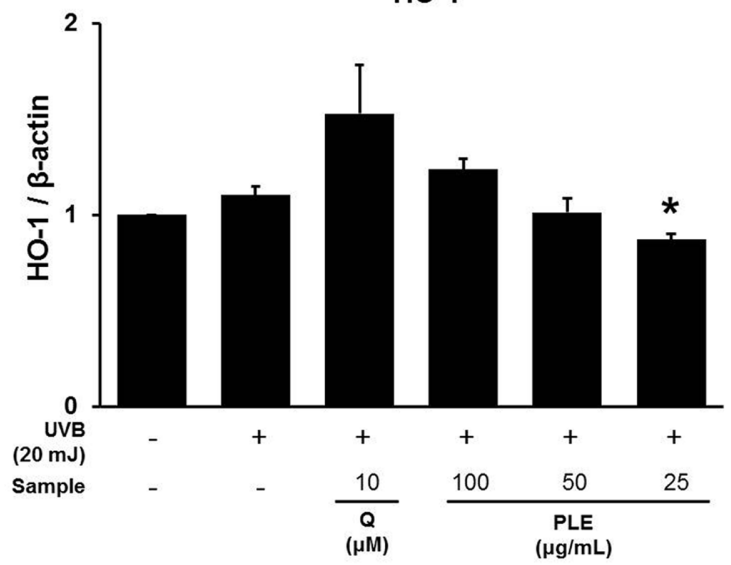

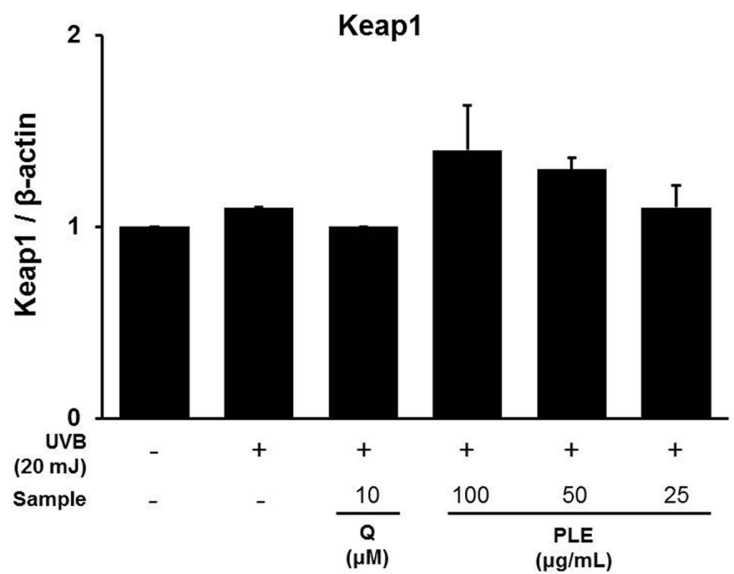

NQO-1

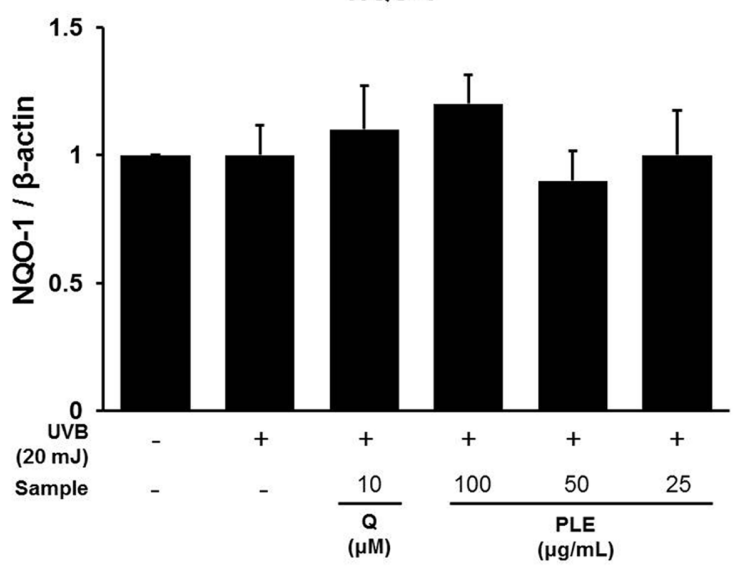

Fig. 5. Expressions of antioxidant enzymes on Pueraria lobata treated HDF.

(A) Expressions of enzymes were examined using Western blotting analysis. (B) The graph above is a densitometry of repeating western blotting three times. Data represent the mean \pm standard error; asterisks indicate significant $(p<0.05)$ changes in expression of Nrf2, HO-1, and NQO- 1 as compared to the UVB-treated group $(0 \mu \mathrm{g} / \mathrm{ml})$. PLE, Pueraria lobata extract treatment group cultured in medium; $\mathrm{Q}$, quercetin group was used as a positive control.

\section{Effect of PLE on Expression of Antioxidant Enzymes}

To investigate the effects of PLE on the expression of a variety of antioxidant enzymes in UVB-irradiated HDF, cells were treated with a number of concentrations of PLE, and incubated for $24 \mathrm{~h}$. After UVB irradiation $\left(20 \mathrm{~mJ} / \mathrm{cm}^{2}\right)$, the expression of protein was examined using western blotting. 
Nrf2, a key transcription regulator of antioxidant enzymes, which regulates the expression of antioxidant enzymes such as NQO-1 and HO-1, binds to the Keap1 protein under normal conditions and is present in the cytoplasm. However, when the amount of oxidative stress in the cell increases, as seen in UVB irradiation, the Keap1 protein is degraded and releases Nrf2, which translocates to the nucleus. Then, Nrf2 mediates the expression of antioxidant enzymes in the ARE region of the gene [19].

As shown in the experimental results (Fig. 5), the overall expression of $\mathrm{Nrf} 2$ was seen to be increased when compared with the control. Interestingly, however, the expression pattern of Nrf2 slightly increased with PLE in a dose-dependent manner (Fig. 5B). The reaction of Nrf2 is very transient, activated by external oxidative stress or antioxidative substances to translocate to the nucleus and activate downstream antioxidant enzymes [19]. Therefore, the expression level of Nrf2 is not considered high because
Nrf2 is degraded after the antioxidant enzyme is expressed after being transferred to the nucleus. The expression of HO-1 and NQO-1 also slightly increased (Figs. 5C and 5D). Therefore, it can be deduced that Nrf2 levels at the initial stage were relatively high, and these translocated to the nucleus and regulated the expression of downstream antioxidant enzymes, and then decreased their levels. This conclusion is consistent with previous findings, where concentrations of Nrf2 were closely dependent on cellular ROS levels and were very finely regulated [26].

\section{Antioxidative Effects of PLE and Correlation with Collagen Synthesis}

Confocal microscopy showed that stimulation of PLE induced the expression of NQO-1, the downstream antioxidant enzyme of Nrf2, and COL1A1, type I collagen (Fig. 6). The nuclei were stained with blue, as shown. The merged image of the $0.1 \%$ PLE-treated group appears
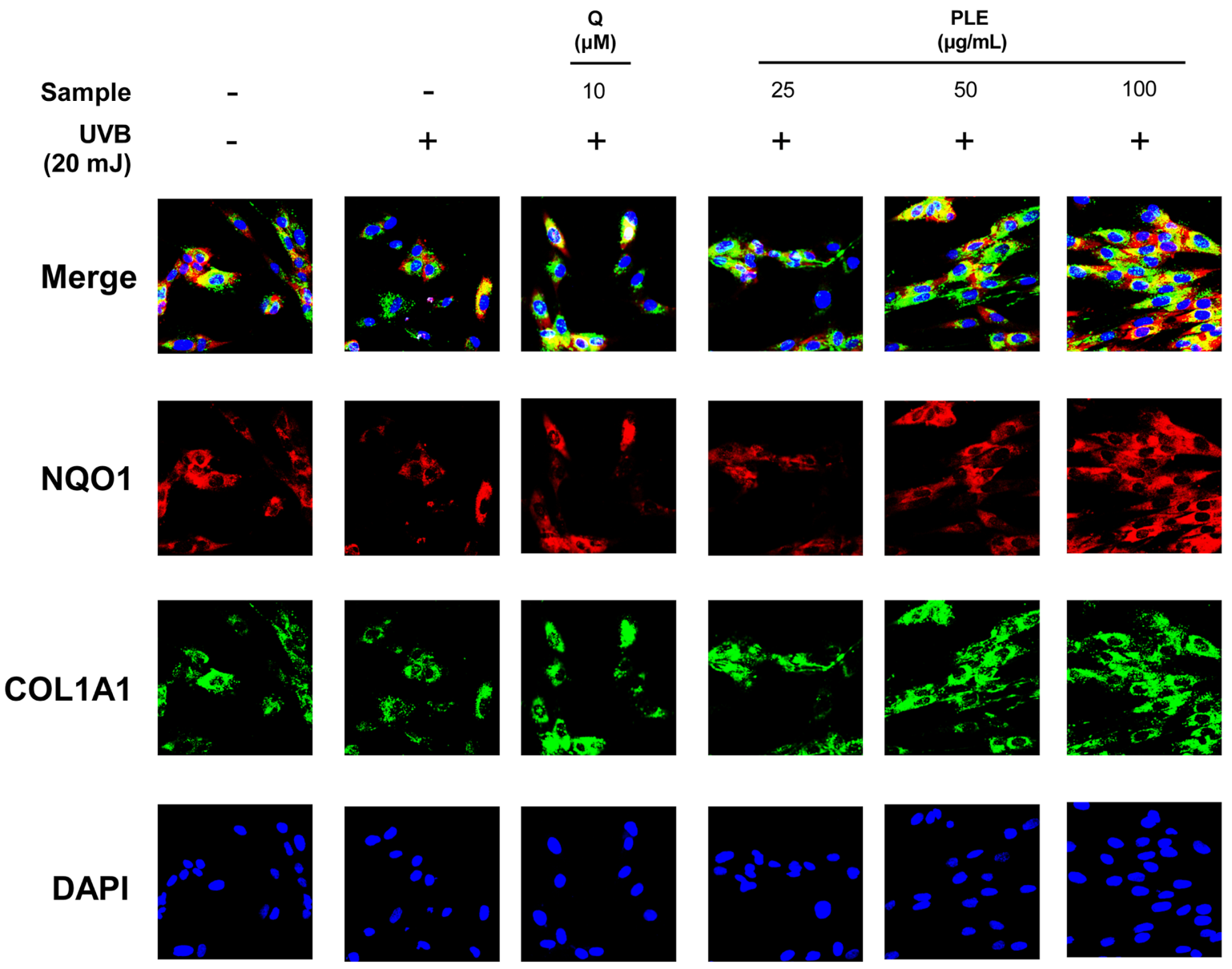

Fig. 6. Confocal microscopy images of NQO-1 (red) and COL1A1 (green) in UVB-irradiated HDF.

PLE, Pueraria lobata extract treatment group cultured in medium; Q, quercetin group used as a positive control; blue indicates DAPI which stains cell nucleus; NQO-1, NAD(P)H: quinone oxidoreductase 1; COL1A1, collagen type 1 alpha 1. 
almost the same as that of the control, which had a good distribution of NQO-1 and COL1A1. Thus, it can be concluded that treatment with PLE regenerated cellular collagen through the activation of NQO-1. Despite the relatively low level of Nrf2 expression in HDF when compared with the control, PLE conclusively demonstrated a strong activity in the reduction of ROS levels in HDF, through the Nrf2 cytoprotective pathway.

PLE reduced HDF intracellular ROS levels via Nrf2mediated downstream antioxidant enzymes, including HO-1 and NQO-1. Furthermore, PLE increased collagen production and maintained HA levels after UVB irradiation, hence inhibiting photoaging in HDF. This study has also provided useful data for the future use of Pueraria montana var. lobata as a potential antioxidant reagent. Previously, a number of studies have been carried out, including skin whitening, use of fermented product, and anti-inflammatory effects of Pueraria lobata on skin [27-29]. In the present study, it was the first in which Pueraria lobata was shown to have the effect of inhibiting photoaging by activating Nrf2, a defense mechanism in the human body.

\section{Acknowledgments}

This work was supported by Bio-Theme Cluster Program of the Korea Industrial Complex Corp. (KICOX) (RDNWIC 17020) and the Basic Science Research Program through the National Research Foundation of Korea (NRF) funded by the the Ministry of Education (NRF-2018R1D1A1B07049077).

\section{Conflict of Interest}

The authors have no financial conflicts of interest to declare.

\section{References}

1. Baumann L. 2007. Skin ageing and its treatment. J. Pathol. 211: 241-251.

2. Debacq-Chainiaux F, Leduc C, Verbeke A, Toussaint O. 2012. UV, stress and aging. Dermatoendocrinol. 4: 236-240.

3. Makrantonaki E, Bekou V, Zouboulis CC. 2012. Genetics and skin aging. Dermatoendocrinol. 4: 280-284.

4. Wlaschek M, Tantcheva-Poor I, Naderi L, Ma W, Schneider LA, Razi-Wolf Z, et al. 2001. Solar UV irradiation and dermal photoaging. J. Photochem. Photobiol. B. 63: 41-51.

5. Ma W, Wlaschek M, Tantcheva-Poor I, Schneider LA, Naderi L, Razi-Wolf Z, et al. 2001. Chronological ageing and photoageing of the fibroblasts and the dermal connective tissue. Clin. Exp. Dermatol. 26: 592-599.
6. Yaar M, Eller MS, Gilchrest BA. 2002. Fifty years of skin aging. J. Investig. Dermatol. Symp. Proc. 7: 51-58.

7. Helfrich YR, Sachs DL, Voorhees JJ. 2008. Overview of skin aging and photoaging. Dermatol. Nurs. 20: 177-183; quiz 184.

8. Fisher GJ, Datta SC, Talwar HS, Wang ZQ, Varani J, Kang S, et al. 1996. Molecular basis of sun-induced premature skin ageing and retinoid antagonism. Nature 379: 335-339.

9. Kim J, Lee C-W, Kim EK, Lee S-J, Park N-H, Kim H-S, et al. 2011. Inhibition effect of Gynura procumbens extract on UVB-induced matrix-metalloproteinase expression in human dermal fibroblasts. J. Ethnopharmacol. 137: 427-433.

10. Kohl E, Steinbauer J, Landthaler M, Szeimies RM. 2011. Skin ageing. J. Eur. Acad. Dermatol. Venereol. 25: 873-884.

11. Pittayapruek P, Meephansan J, Prapapan O, Komine M, Ohtsuki M. 2016. Role of matrix metalloproteinases in photoaging and photocarcinogenesis. Int. J. Mol. Sci. 17(6).

12. Espinosa-Diez C, Miguel V, Mennerich D, Kietzmann T, Sanchez-Perez P, Cadenas S, et al. 2015. Antioxidant responses and cellular adjustments to oxidative stress. Redox. Biol. 6: 183-197.

13. Loft S, Poulsen HE. 1996. Cancer risk and oxidative DNA damage in man. J. Mol. Med (Berl). 74: 297-312.

14. Rabilloud T, Chevallet M, Luche S, Leize-Wagner E. 2005. Oxidative stress response: a proteomic view. Expert Rev. Proteomics. 2: 949-956.

15. Zhang DD. 2006. Mechanistic studies of the Nrf2-Keap1 signaling pathway. Drug Metab. Rev. 38: 769-789.

16. Giudice A, Arra C, Turco MC. 2010. Review of molecular mechanisms involved in the activation of the Nrf2-ARE signaling pathway by chemopreventive agents. Methods Mol. Biol. 647: 37-74.

17. Kumar H, Kim IS, More SV, Kim BW, Choi DK. 2014. Natural product-derived pharmacological modulators of Nrf2/ARE pathway for chronic diseases. Nat. Prod. Rep. 31: 109-139.

18. Dinkova-Kostova AT, Talalay P. 2008. Direct and indirect antioxidant properties of inducers of cytoprotective proteins. Mol. Nutr. Food Res. 52 Suppl 1: S128-138.

19. Lee JH, Khor TO, Shu L, Su ZY, Fuentes F, Kong AN. 2013. Dietary phytochemicals and cancer prevention: Nrf2 signaling, epigenetics, and cell death mechanisms in blocking cancer initiation and progression. Pharmacol. Ther. 137: 153-171.

20. He CH, Gong P, Hu B, Stewart D, Choi ME, Choi AM, et al. 2001. Identification of activating transcription factor 4 (ATF4) as an Nrf2-interacting protein. Implication for heme oxygenase-1 gene regulation. J. Biol. Chem. 276: 20858-20865.

21. Li N, Alam J, Venkatesan MI, Eiguren-Fernandez A, Schmitz D, Di Stefano E, et al. 2004. Nrf2 is a key transcription factor that regulates antioxidant defense in macrophages and epithelial cells: protecting against the proinflammatory and oxidizing effects of diesel exhaust chemicals. J. Immunol. 173: 3467-3481. 
22. Siegel D, Bolton EM, Burr JA, Liebler DC, Ross D. 1997. The reduction of alpha-tocopherolquinone by human $\mathrm{NAD}(\mathrm{P}) \mathrm{H}$ : quinone oxidoreductase: the role of alpha-tocopherolhydroquinone as a cellular antioxidant. Mol. Pharmacol. 52: 300-305.

23. Bebrevska L, Foubert K, Hermans N, Chatterjee S, Van Marck E, De Meyer G, et al. 2010. In vivo antioxidative activity of a quantified Pueraria lobata root extract. J. Ethnopharmacol. 127: 112-117.

24. Park HJ, Cho DH, Kim HJ, Lee JY, Cho BK, Bang SI, et al. 2009. Collagen synthesis is suppressed in dermal fibroblasts by the human antimicrobial peptide LL-37. J. Invest. Dermatol. 129: 843-850.

25. Pawlikowska-Pawlęga B, Ignacy Gruszecki W, Misiak L, Paduch R, Piersiak T, Zarzyka B, et al. 2007. Modification of membranes by quercetin, a naturally occurring flavonoid, via its incorporation in the polar head group. Biochim. Biophys. Acta 1768: 2195-2204.

26. Matzinger M, Fischhuber K, Heiss EH. 2018. Activation of Nrf2 signaling by natural products-can it alleviate diabetes? Biotechnol. Adv. 36: 1738-1767.

27. Kwon JE, Lim J, Bang I, Kim I, Kim D, Kang SC. 2019. Fermentation product with new equol-producing Lactobacillus paracasei as a probiotic like product candidate for prevention of skin and intestinal disorder. J. Sci. Food Agric. doi: 10.1002/ jsfa.9648. [Epub ahead of print]

28. Lee J-H, Jeon Y-D, Lee Y-M, Kim D-K. 2018. The suppressive effect of puerarin on atopic dermatitis-like skin lesions through regulation of inflammatory mediators in vitro and in vivo. Biochem. Biophys. Res. Commun. 498: 707-714.

29. Han E, Chang B, Kim D, Cho H, Kim S. 2015. Melanogenesis inhibitory effect of aerial part of Pueraria thunbergiana in vitro and in vivo. Arch. Dermatol. Res. 307: 57-72. 